\title{
The pressure-temperature phase diagram of metacetamol and its comparison to the phase diagram of paracetamol
}

\author{
M. Barrio ${ }^{1}$, J. Huguet ${ }^{1}$, I. B. Rietveld ${ }^{2, *}$, B. Robert ${ }^{3}$, R. Céolin ${ }^{1,4}$, J.Ll. Tamarit ${ }^{1}$ \\ ${ }^{1}$ Departament de Física, ETSEIB, Universitat Politècnica de Catalunya, Diagonal 647, 08028 \\ Barcelona, Catalonia, Spain \\ ${ }^{2}$ Laboratoire de Chimie Physique, CAMMAT, Faculté de Pharmacie, Université Paris \\ Descartes, 4 avenue de l’Observatoire, 75006 Paris, France \\ ${ }^{3}$ Sanofi R\&D, Lead Generation \& Compound Realization/Analytical Sciences/Solid State \\ group, 13 quai Jules Guesde, F-94400 Vitry sur Seine, France \\ ${ }^{4}$ LETIAM, EA7357, IUT Orsay, Université Paris Sud, rue Noetzlin, 91405 Orsay Cedex, \\ France \\ * Corresponding author: ivo.rietveld@parisdescartes.fr
}




\begin{abstract}
Understanding the polymorphic behavior of active pharmaceutical ingredients is important for formulation purposes and for regulatory reasons. Metacetamol is an isomer of paracetamol and it similarly exhibits polymorphism. In the present paper, it has been found that one of the polymorphs of metacetamol is only stable under increased pressure, which has led to the conclusion that metacetamol like paracetamol is a monotropic system under ordinary (= laboratory) conditions and that it becomes enantiotropic under pressure with the I-II-L triple point coordinates for metacetamol $T_{\text {I-II-L }}=535 \pm 10 \mathrm{~K}$ and $P_{\text {I-II-L }}=692 \pm 70 \mathrm{MPa}$. However, whereas for paracetamol the enantiotropy under pressure can be foreseen, because the metastable polymorph is denser, in the case of metacetamol this is not possible, as the metastable polymorph is less dense than the stable one. The existence of the stability domain for the less dense polymorph of metacetamol, can only be demonstrated by the construction of the topological phase diagram as presented in this paper. It is a delicate interplay between the specific volume differences and the enthalpy differences causing the stability domain of the less dense polymorph to be sandwiched between the denser polymorph and the liquid. It is a characteristic that metacetamol shares with bicalutamide and fluoxetine nitrate.
\end{abstract}

Keywords:

Calorimetry, crystal polymorphism, physical characterization, phase behavior, topological phase diagram, thermodynamics, solid state, thermal expansion, physical stability 


\section{Introduction}

\subsection{Metacetamol}

Metacetamol is an isomer of the well-known drug paracetamol and is also known as 3acetamidophenol or $N$-(3-hydroxyphenyl)acetamide. The chemical structure is provided in Figure 1. Its crystalline dimorphism has been demonstrated by McGregor et al, who recently published the structure of a new polymorph, form II, which is monoclinic with space group $\mathrm{P} 2_{1}{ }^{1}$. They also confirmed the structure of the known polymorph, form I, which is orthorhombic with space group Pna2 $2_{1}{ }^{1,2}$<smiles>CC(=O)Nc1ccc(O)cc1</smiles>

Figure 1. Chemical structure of metacetamol (left-hand side) ( $\mathrm{N}$-(3hydroxyphenyl)acetamide), $\mathrm{C}_{8} \mathrm{H}_{9} \mathrm{~N}_{2} \mathrm{O}_{2}, \mathrm{M}=151.16 \mathrm{~g} \mathrm{~mol}^{-1}$ and for comparison on the righthand side, the chemical structure of paracetamol

The present study has been carried out in the framework of a more general study on the pressure-temperature behavior of polymorphism in small organic molecules and in particular in active pharmaceutical ingredients (APIs). Often, polymorphism of active ingredients is tested by a few calorimetric measurements in a differential thermal analyzer (DSC) and transition temperatures are reported with the accuracy of the equipment stated in the manual. In an ideal world this approach would certainly be viable, but often the system exhibits more complicated behavior, which only becomes apparent when multiple measurements at multiple heating rates are carried out. The API may be decomposing or the solid-solid transition of an enantiotropic system may depend on the heating rate of the DSC measurements. ${ }^{3-5}$ It implies that it is often wise to establish the best possible representation of the phase behavior of an API including its behavior under pressure. The latter parameter is important, because thermodynamics is consistent. The phase behavior under pressure must coincide with that observed under "ordinary conditions" (i.e. conditions where the system is free to set its own pressure as in a DSC for example). High-pressure phase transition measurements can therefore be used for verification. In other cases, high-pressure data may even be used to find transition temperatures that are not observed by measurements under "ordinary conditions", as was the case for paracetamol ${ }^{6,7}$ and other systems. ${ }^{5,8}$ Measurements will always depend on kinetic factors (also high-pressure measurements for that matter, but the response of the system may be different), but the thermodynamic behavior of a system only depend on the differences in Gibbs energy between the phases. The most obvious reason to use high-pressure measurements is to study the response of an API to pressure, because during tableting and grinding it will be subjected to changing pressure. In particular for monotropic systems such as paracetamol the fact that a second polymorph exists, but with an unknown stability domain, essentially obliges the scientist in preformulation to investigate, where the solid-solid transition is located as a function of pressure and temperature, because one would like to avoid a sudden transition into the metastable form simply by processing the API under pressure. 
The objective of this paper was to establish the topological and experimental pressuretemperature (P-T) phase diagram of the dimorphism of metacetamol. Data at ordinary pressure and experimental measurements under pressure have been obtained. In the case of its isomer paracetamol, the topological approach had led to the correct prediction of the experimental pressure-temperature phase diagram that was obtained a few years later. ${ }^{6,7}$ Because paracetamol and metacetamol are closely related isomers, a comparison will be made between the phase diagrams of the two isomers.

\subsection{Available data from the literature}

The melting point of form I and its melting enthalpy have been determined by Perlovich et al.: ${ }^{9} T_{\mathrm{I} \rightarrow \mathrm{L}}=416.2 \mathrm{~K}, \Delta_{\mathrm{I} \rightarrow \mathrm{L}} H=24.6 \mathrm{~kJ} \mathrm{~mol}^{-1}\left(=163 \mathrm{~J} \mathrm{~g}^{-1}\right)$. They determined its vapor pressure too in the range from $344.5 \mathrm{~K}$ to $397 \mathrm{~K}$, which can be summarized as:

$\ln P_{\mathrm{I} \rightarrow \mathrm{V}} / \mathrm{Pa}=(33.0 \pm 0.4)-(13188 \pm 138) /(T / \mathrm{K})$

Unfortunately, McGregor et al, who used differential scanning calorimetry (DSC) to determine the temperatures of fusion of forms I and II and reported onsets at $420.5 \mathrm{~K}$ and 400 $\mathrm{K}$, respectively, did not mention the melting enthalpies. ${ }^{1}$ However, they did provide the recorded DSC curves on heating and on reheating of the same specimen and therefore the surface areas of the melting peaks of the two forms could be determined. The surface ratio of 1.19 equals the ratio $\Delta_{\mathrm{I} \rightarrow \mathrm{L}} H / \Delta_{\mathrm{II} \rightarrow \mathrm{L}} H$, leading to $\Delta_{\mathrm{II} \rightarrow \mathrm{L}} H=137 \mathrm{~J} \mathrm{~g}^{-1}$ using the known value of $\Delta_{\mathrm{I} \rightarrow \mathrm{L}} H$ previously found by Perlovich et al. ${ }^{9}$ A summary of the calorimetric results can be found in Table 1. 
Table 1. Crystallographic and calorimetric data available in the literature for the two known polymorphs of metacetamol

\begin{tabular}{cccc}
\hline & $\begin{array}{c}\text { Form I, } \\
\text { Orthorhombic, } \\
\text { Pna2 }\end{array}$ & $\begin{array}{c}\text { Form II, } \\
\text { monoclinic, } \\
\text { P2 } \mathbf{1}_{\mathbf{1}}\end{array}$ & Ref \\
\hline Temperature $/ \mathrm{K}$ & $v_{\mathrm{I}} / \mathrm{cm}^{3} \mathrm{~g}^{-1}$ & $v_{\mathrm{II}} / \mathrm{cm}^{3} \mathrm{~g}^{-1}$ & \\
\hline 120 & 0.72586 & 0.72943 & 1 \\
\hline 298 & - & 0.75453 & 1 \\
\hline 293 & 0.75635 & - & 2 \\
\hline
\end{tabular}

\begin{tabular}{cccc} 
Polymorph & $\mathrm{T}_{\text {fus }} / \mathrm{K}$ & $\Delta_{\text {fus }} \mathrm{H} / \mathrm{J} \mathrm{g} \mathrm{g}^{-1}$ & Ref \\
\hline Form I & 416.2 & 162.74 & 1,9 \\
& 420.5 & - & \\
\cline { 2 - 4 } & $420.6 \pm 1.0$ & $190.6 \pm 4.6$ & This work \\
\hline Form II & 400 & $137^{\mathrm{a}}$ & 1 \\
\cline { 2 - 4 } & $399.4 \pm 1.0$ & $156.6 \pm 4.5$ & This work \\
\hline
\end{tabular}

a estimated with the ratio between the areas of the two melting peaks in ref 1 .

The specific volume for both polymorphs can be found in Table 1. Using the limited temperature dependent data and assuming a linear relationship, the following two expressions for the specific volume as a function of the temperature can be found:

$v_{\mathrm{I}} / \mathrm{cm}^{3} \mathrm{~g}^{-1}=0.70471+0.00017624 \cdot \mathrm{T} / \mathrm{K}$

$v_{\text {II }} / \mathrm{cm}^{3} \mathrm{~g}^{-1}=0.71251+0.00014101 \cdot \mathrm{T} / \mathrm{K}$

From these two expressions, also the expansivity coefficients $\alpha_{\mathrm{V}}$ can be obtained $(v=$ $v_{0} \cdot\left(1+\alpha_{\mathrm{V}} \cdot T\right)$. For $\alpha_{\mathrm{V}, \mathrm{I}}$, it leads to $2.50 \times 10^{-4} \mathrm{~K}^{-1}$ and for $\alpha_{\mathrm{V}, \text { II }} 1.98 \times 10^{-4} \mathrm{~K}^{-1}$ can be found. Both expansivity coefficients are close to the average value of about $2 \times 10^{-4} \mathrm{~K}^{-1}$ found for molecular solids $^{10-19}$ and active pharmaceutical ingredients. ${ }^{20-23}$ However, a closer study of the two expressions 2 and 3 and the data in Table 1 demonstrates that although form I appears to be denser at low temperature, it exhibits the largest thermal expansion $\left(\alpha_{\mathrm{V}, \mathrm{I}}>\alpha_{\mathrm{V}, \mathrm{II}}\right)$ and at room temperature its density is actually less than that of form II. This creates a thermodynamic dilemma, because two polymorphs with distinct internal energies due to the different interactions between the molecules can hardly lead to the same specific volume without encountering a discontinuity (i.e. phase equilibrium). This thermodynamic inconsistency is most likely due to the different X-ray diffraction equipment used for the measurement at 293 K.

If one were to construct the topological pressure-temperature phase diagram of metacetamol, it will be necessary to determine the volume inequality between the two phases unequivocally. In addition, reliable calorimetric data are a necessity and if possible a comparison with an experimentally obtained P-T phase diagram would be welcome.

\section{Materials and Methods}

Metacetamol was purchased from Sigma-Aldrich with a purity higher than $97 \%$. It was used as such after verification by X-ray diffraction that all peaks could be ascribed to polymorph I. 
High-resolution X-ray powder diffraction measurements using the Debye-Scherrer geometry and transmission mode were carried out with a vertically mounted INEL cylindrical position sensitive detector (CPS-120) with 4096 channels $\left(0.029^{\circ}-2 \theta\right.$ angular step). Monochromatic $\mathrm{Cu}-\mathrm{K} \alpha_{1} \quad(\lambda=1.54056 \AA)$ radiation was selected by means of an asymmetrically focusing incident-beam curved quartz monochromator. The generator power was set to $35 \mathrm{kV}$ and $35 \mathrm{~mA}$. Measurements as a function of temperature were conducted by means of a liquid nitrogen 700 series Cryostream Cooler from Oxford, UK Cryosystems. External calibration by means of the cubic phase $\mathrm{Na}_{2} \mathrm{Ca}_{3} \mathrm{Al}_{2} \mathrm{~F}_{4}$ and cubic spline fitting was used to convert the measurement channels into $2 \theta$. Peak positions were determined using pseudo-Voigt fitting and lattice parameters were refined using the least-squares option of the FullProf suite package. $^{24,25}$

The samples were introduced in Lindemann capillaries with $0.5-\mathrm{mm}$ diameter, which rotated perpendicularly to the X-ray beam during the experiments to improve the averaging over the crystallite orientations. Before data acquisition, each sample was allowed to equilibrate at the temperature of measurement for not less than $10 \mathrm{~min}$, and each acquisition time was at least one hour. Patterns were recorded on heating in the temperature range from $100 \mathrm{~K}$ up to the melting point for each polymorph.

Calorimetric data were obtained with a Q100 thermal analyzer from TA Instruments (New Castle, USA). Specimens with sample masses between 2 and $10 \mathrm{mg}$ were weighed using a microbalance sensitive to $0.01 \mathrm{mg}$ and sealed in aluminum pans.

High-pressure differential thermal analysis (HP-DTA) was carried out with an inhouse constructed HP-DTA similar to the apparatus built by Würflinger ${ }^{26}$ operating between 200-473 K and 0-300 MPa. Melting temperatures as a function of a fixed pressure were determined of specimens mixed with an inert perfluorinated liquid (Galden, from Bioblock Scientifics, Illkirch, France) to ascertain that in-pan volumes were free from residual air. Because loss of sample during the filling process cannot be controlled, the precise mass in the high-pressure capsule is not known. Perfluorinated liquid did not interact with metacetamol as demonstrated by DSC measurements carried out on a Galden + metacetamol mixture using the TA Instruments Q100.

\section{Results}

The temperature and enthalpy of fusion of form I were found to be $420.6 \pm 1.0 \mathrm{~K}$ and $190.6 \pm 4.6 \mathrm{~J} \mathrm{~g}^{-1}$ respectively. They are the mean values from 7 independent DSC measurements at heating rates of 2 and $5 \mathrm{~K} \mathrm{~min}^{-1}$. An example of the melting peak of form I is marked by a1 in Figure 2. On cooling, one observes randomly either the recrystallization to form II, marked by a2 in Figure 2, or the formation of a glass with a $T_{\mathrm{g}}$ equal to $293 \mathrm{~K}$ (midpoint) and shown in the inset in Figure 2. On heating the same sample for a second time, melting of form II, marked by a3 in Figure 2, or recrystallization of the supercooled liquid, marked by b1 in Figure 2, subsequently followed by the melting of form II, marked by b2 in Figure 2, are observed.

The temperature and enthalpy of fusion of form II were found to be $399.4 \pm 1 \mathrm{~K}$ and $156.6 \pm 4.5 \mathrm{~J} \mathrm{~g}^{-1}$ respectively. They are the mean values from 6 independent DSC measurements at heating rates of 2 and $5 \mathrm{~K} \mathrm{~min}^{-1}$.

Although the enthalpy values are higher than those reported by Perlovitch et al, ${ }^{9}$ the ratio $\Delta_{\mathrm{I} \rightarrow \mathrm{L}} H / \Delta_{\mathrm{II} \rightarrow \mathrm{L}} H$ equals 1.22 , which is close to the value of 1.19 found in the article by McGregor et al. ${ }^{1}$

During one of the second heating runs by DSC, after the recrystallization into form II marked by c1 in Figure 2 serendipitously an additional exothermic effect was observed marked by c2, which was ascribed to the conversion of form II into form I. It can be seen that after this conversion form I melts at its ordinary melting point (c3 in Figure 2). The enthalpy 
involved in the observed conversion was $-24 \mathrm{~J} \mathrm{~g}^{-1}$. The enthalpy difference between the two polymorphs can also be obtained from the two melting points, neglecting the difference in the specific heats. It leads to an enthalpy difference, $\Delta_{\mathrm{II} \rightarrow \mathrm{I}} H$, of $-34 \pm 9 \mathrm{~J} \mathrm{~g}^{-1}$.

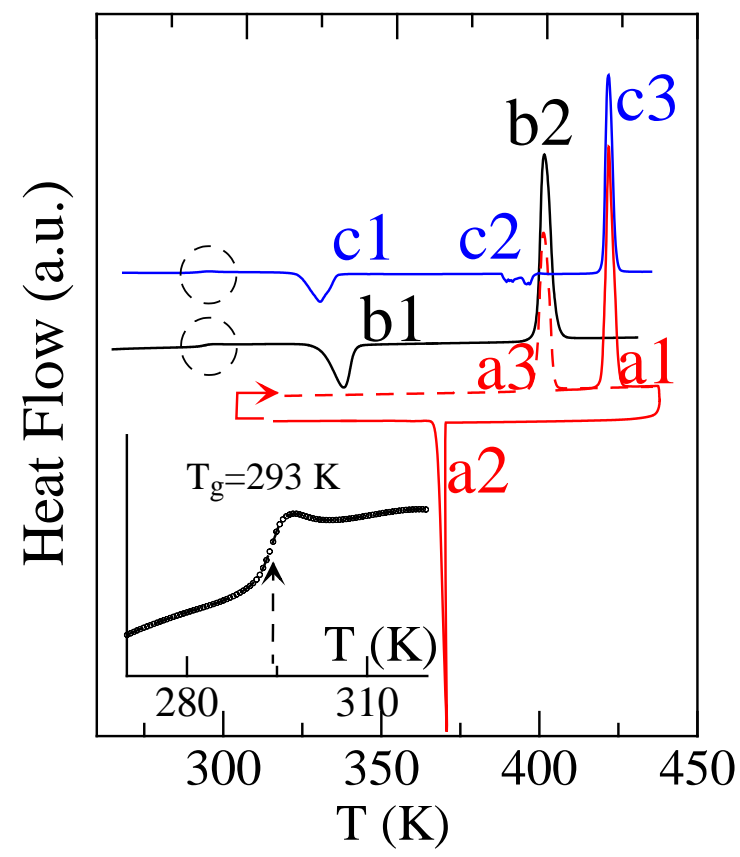

Figure 2. Thermograms obtained by differential scanning calorimetry of samples of form I of metacetamol. Red curve, marked by a: melting of form I (a1), which after cooling from the liquid phase leads to recrystallization (a2) into form II, which melts on the second heating run (a3). Black curve, marked by b: heating curve obtained after fast cooling of the melt giving rise to the glass transition (small black circles, see inset), followed by recrystallization into form II (b1) and by fusion of form II (b2). The blue curve was obtained in the same way as the black curve; however, the exothermic transition from form II to form I (c2) appeared serendipitously, as demonstrated by the subsequent melting of form I (c3) at its ordinary melting point.

$\mathrm{X}$-ray diffraction patterns obtained as a function of temperature were fitted against the published structures of the two polymorphs using FullProf. ${ }^{24,25}$ Examples of the X-ray patterns obtained at room temperature have been provided in Figure 3. The lattice parameters as a function of temperature were used to determine the specific volumes from $100 \mathrm{~K}$ up to the melting temperature of each polymorph, which have been plotted in Figure 4. The result in Figure 4 clearly demonstrates that the specific volume of form II is larger than that of form I at any given temperature. Therefore, the results reported in the literature and listed in Table 1 may suffer lack of accuracy, in particular for form II. The specific volumes were fitted as a function of temperature using the least-squares method leading to the following two equations for forms I and II, respectively:

$$
\begin{aligned}
& v_{\mathrm{I}} / \mathrm{cm}^{3} \mathrm{~g}^{-1}=0.7112( \pm 0.0005)+1.36( \pm 0.04) \times 10^{-4} \mathrm{~T} / \mathrm{K}+7.3( \pm 0.7) \times 10^{-8} \mathrm{~T}^{2} / \mathrm{K} \quad \mathrm{R}^{2}=0.9999 \\
& v_{\mathrm{II}} / \mathrm{cm}^{3} \mathrm{~g}^{-1}=0.7222( \pm 0.0053)+1.0( \pm 0.4) \times 10^{-4} \mathrm{~T} / \mathrm{K}+1.1( \pm 0.8) \times 10^{-7} \mathrm{~T}^{2} / \mathrm{K} \quad \mathrm{R}^{2}=0.996
\end{aligned}
$$



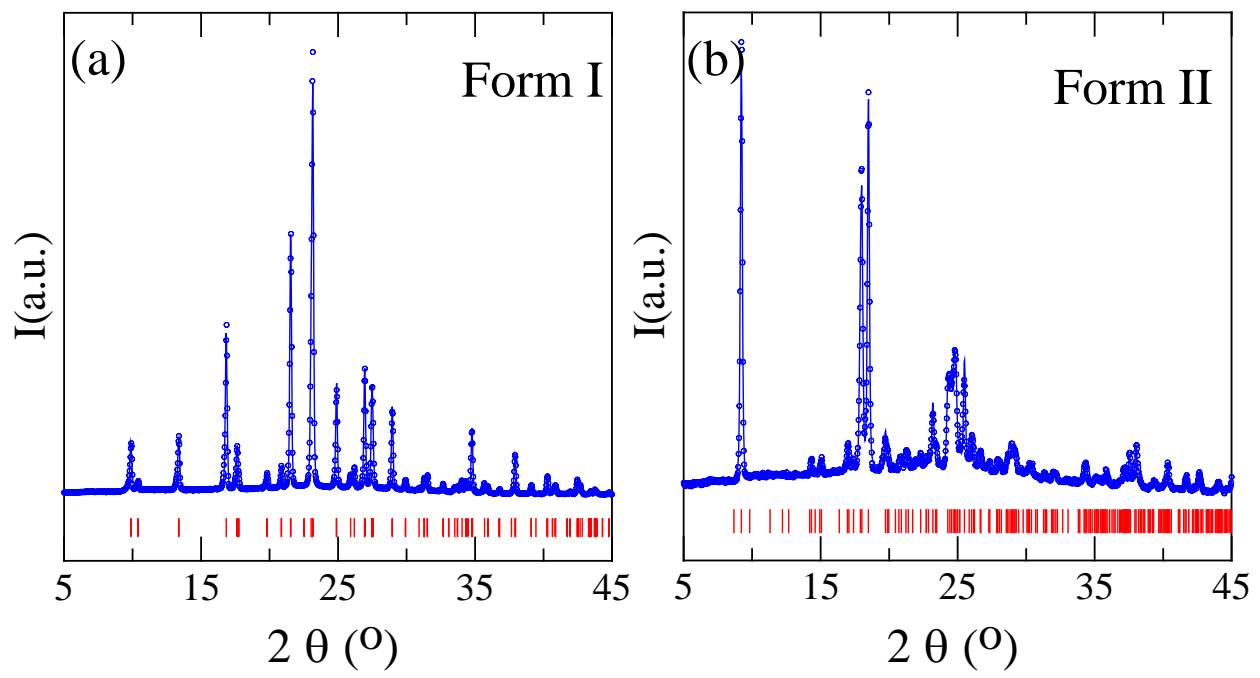

Figure 3. Experimental (points) and fitted (lines) diffraction patterns along with the calculated Bragg peak positions (red vertical bars) for (a) the orthorhombic Pna2 ${ }_{1}$ form I and (b) the monoclinic $P 2{ }_{1}$ form II.

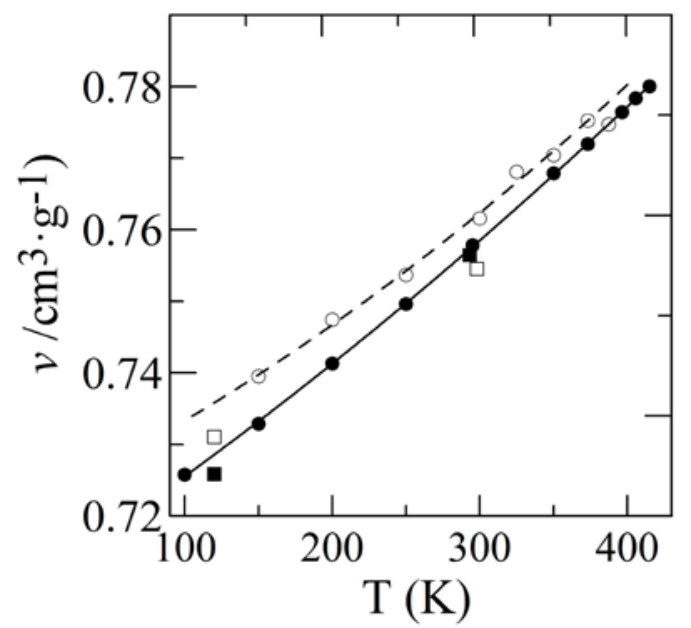

Figure 4. The specific volume of metacetamol as a function of temperature for form I (solid circles) and form II (open circles). Literature data reported in Table 1 have been plotted too: solid squares for form I and open squares for form II.

High-pressure differential thermal analysis (HP-DTA) was carried out for a series of pressures for form I as well as for form II. Several examples of high-pressure differential thermal analysis curves are presented in Figure 5 for both polymorphs. Using the onset temperatures of the melting curves, the melting temperature of each polymorph as a function of the pressure can be fitted to a straight line:

$$
\begin{array}{ll}
P_{\mathrm{I} \rightarrow \mathrm{L}} / \mathrm{MPa}=5.83( \pm 0.13) \mathrm{T} / \mathrm{K}-2430( \pm 59) & \mathrm{R}^{2}=0.999 \\
P_{\mathrm{II} \rightarrow \mathrm{L}} / \mathrm{MPa}=4.92( \pm 0.11) \mathrm{T} / \mathrm{K}-1942( \pm 28) & \mathrm{R}^{2}=0.997
\end{array}
$$

The HP-DTA results demonstrate that the melting temperatures of the two polymorphs approach each other on increasing pressure as the slope of the lower melting form II is shallower than the slope of the higher melting form I. The convergence of the melting curves can also be seen in Figure 6, where the melting onsets have been plotted as a function of temperature and pressure for both forms. 

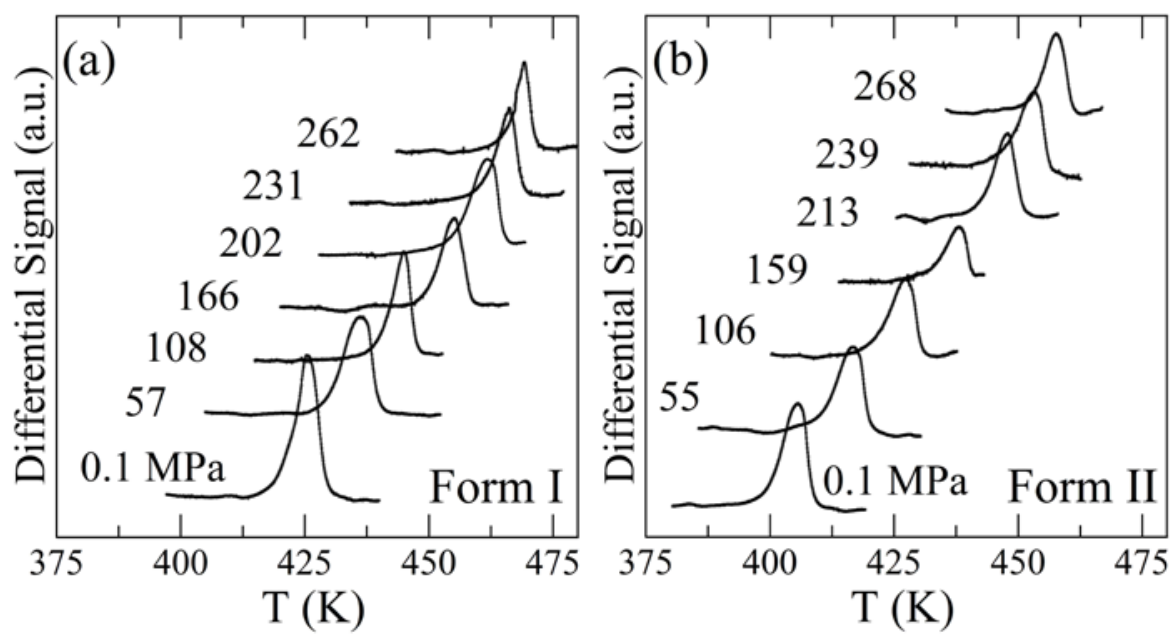

Figure 5. High-pressure differential thermal analysis curves of the melting transition of (a) form I and (b) form II at different hydrostatic pressures (in MPa)

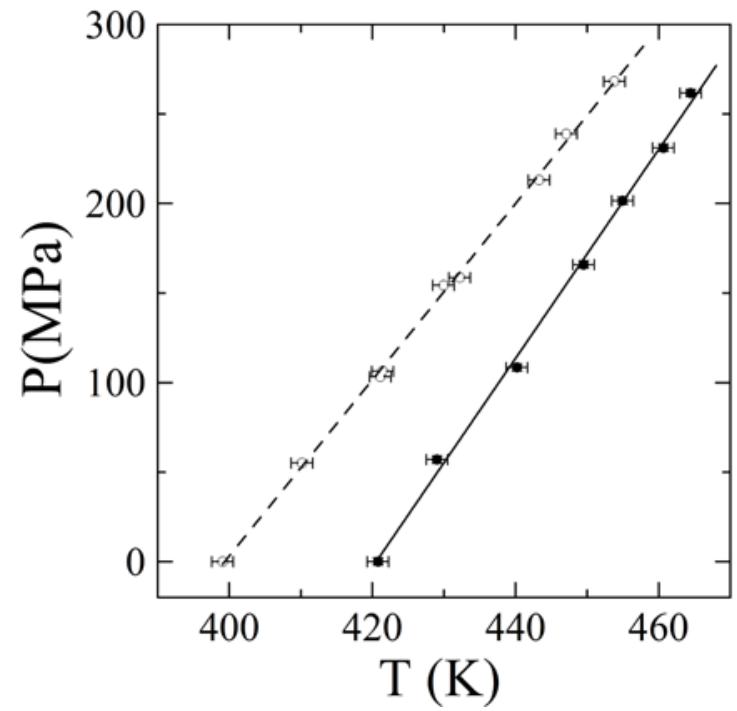

Figure 6. Melting curves of the two polymorphs (form I: solid circles; form II: open circles) as a function of pressure.

\section{Discussion}

To construct the pressure-temperature phase diagram of the dimorphism of metacetamol, it is necessary to determine the coordinates of the four triple points that exist for a dimorphic system: I-II-L, I-II-V, I-L-V, and II-L-V (with V the vapor phase). ${ }^{27,28}$ The melting points in an ordinary DSC are generally obtained in the presence of a vapor phase, because the capsules are never completely filled. It implies that melting points can be considered triple points with the vapor pressure of the molecule being the pressure coordinate. In the case of the triple point I-II-L both melting equilibria I-L and II-L must coincide. Its coordinates can be obtained by extrapolating the expressions 6 and 7 considering that melting equilibria are generally rather straight lines over a considerable pressure range. Using this approach the I-II-L triple point coordinates are found to be $T_{\mathrm{I}-\mathrm{II}-\mathrm{L}}=535 \pm 10 \mathrm{~K}$ and $P_{\mathrm{I}-\mathrm{II}-\mathrm{L}}=$ $692 \pm 70 \mathrm{MPa}$. This point is marked by $\mathrm{O} 4$ in Figure 7, the topological phase diagram.

As mentioned above, the triple point coordinates of I-L-V consist of the melting temperature of form I determined by DSC and its vapor pressure of metacetamol at the melting point, which has been determined by Perlovich et al. ${ }^{9}$ Thus using the melting point determined in the present study $T_{\mathrm{I}-\mathrm{L}-\mathrm{V}}=421 \mathrm{~K}$ and using this temperature in the sublimation 
pressure equation by Perlovich et al. (eq. 1) a triple point pressure, $P_{\mathrm{I}-\mathrm{L}-\mathrm{V}}$ of $5.2 \pm 1.0 \mathrm{~Pa}$ is found.

For the pressure of the triple point II-L-V, the sublimation pressure at the melting point of form II needs to be known. This can either be obtained through the I-II transition point, where both vapor pressures need to be the same, but this temperature has not been determined yet. The second approach is to determine the vapor pressure of the liquid phase through the melting point of form I. Both approaches make use of thermodynamic cycles or Hess's law. From eq. 1 it is known that the sublimation enthalpy of form I must equal 13188 $\mathrm{R}$ (with $\mathrm{R}=8.3145 \mathrm{~J} \mathrm{~K}^{-1} \mathrm{~mol}^{-1}$ ), which equals 121 109.7 $\pm 1,2 \mathrm{~kJ} \mathrm{~mol}^{-1}$. The melting enthalpy of form I equals $190.6 \mathrm{~J} \mathrm{~g}^{-1}\left(28.8 \mathrm{~kJ} \mathrm{~mol}^{-1}\right)$. Because the vapor phase is the same at the melting equilibrium, its energy content is also the same. Therefore the difference in energy content between the vapor phase and the liquid phase is $109.7-28.8=80.9 \pm 1,9 \mathrm{~kJ} \mathrm{~mol}^{-1}$. The vapor pressure at the melting point is also the same for form I and the liquid, which allows to determine the fitting constant for the expression of the vapor pressure of the liquid leading to the expression:

$\ln P_{\mathrm{L} \rightarrow \mathrm{V}} / \mathrm{Pa}=(24.8 \pm 2.8)-(80850 \pm 1900) /(R T)$

As the melting point of form II is known to be $399.4 \mathrm{~K}$ and because the vapor pressure of the liquid phase and of form II must be the same, the triple point pressure can be calculated with eq. 8 and it equals $1.5 \pm 0.5 \mathrm{~Pa}$.

The only missing triple point coordinates are those of triple point I-II-V, which is in fact the solid-solid transition between form I and form II. Considering that the enthalpy difference between the two solids going from form II to form I was found to be negative and the observation that form II melts at a lower temperature than form I, the I-II-V triple point must lie above the two melting points.

By calculating the slope of the I-II equilibrium, it can be used in combination with the I-II-L triple point to determine the position of the I-II equilibrium curve. The slope is given by the Clapeyron equation:

$$
\mathrm{d} P / \mathrm{d} T=\Delta s / \Delta v=\Delta h / T \Delta v
$$

with $\Delta s$ the specific entropy difference between two phases and $\Delta h$ the specific enthalpy.

From Table 1 the specific entropy of fusion for both solids can be calculated based on the data obtained in the present experimental study: $\Delta s_{\mathrm{I} \rightarrow \mathrm{L}}=0.453 \pm 0.012 \mathrm{~J} \mathrm{~g}^{-1} \mathrm{~K}^{-1}$ and $\Delta s_{\mathrm{II} \rightarrow \mathrm{L}}$ $=0.392 \pm 0.012 \mathrm{~J} \mathrm{~g}^{-1} \mathrm{~K}^{-1}$. Thus the specific entropy change related to the phase change of form I into II equals $\Delta s_{\mathrm{I} \rightarrow \mathrm{II}}=\Delta s_{\mathrm{I} \rightarrow \mathrm{L}}-\Delta s_{\mathrm{II} \rightarrow \mathrm{L}}=0.061 \pm 0.012 \mathrm{~J} \mathrm{~g}^{-1} \mathrm{~K}^{-1}$. In addition, the change in specific volume can be estimated using the experimental results in this paper. At the melting point of form I, the difference in volume between the two phases can be calculated to be $\Delta v_{\mathrm{I} \rightarrow \mathrm{II}}=0.0024 \pm 0.0006 \mathrm{~cm}^{3} \mathrm{~g}^{-1}$. At the I-II-L triple point temperature of $535 \mathrm{~K}$, the volume difference has become $0.0023 \pm 0.0006 \mathrm{~cm}^{3} \mathrm{~g}^{-1}$. This value is low, but rather consistent over a large temperature range. Using the latter value for the volume change, because the I-II-L triple point is part of the I-II equilibrium line, the slope for the I-II equilibrium can be calculated. This leads to the value of $26 \pm 8 \mathrm{MPa} \mathrm{K}^{-1}$ for $\mathrm{d} P / \mathrm{d} T_{\text {I-II. }}$ Even using the measured value of $-24 \mathrm{Jg}^{-1}$ for the transition of form II into form I at about $380 \mathrm{~K}$, leads to an entropy change of $\Delta s_{\mathrm{I} \rightarrow \mathrm{II}}=0.063 \mathrm{~J} \mathrm{~g}^{-1} \mathrm{~K}^{-1}$ (positive, because the transition is taken in the inverse direction) and thus to a slope of $\approx 27 \mathrm{MPa} \mathrm{K}^{-1}$, which is very similar.

Involving the results of eqs. 6 and 7 , the following inequality can be observed: $\mathrm{d} P / \mathrm{d} T_{\mathrm{I}}$ II $>\mathrm{d} P / \mathrm{d} T_{\text {I-L }}>\mathrm{d} P / \mathrm{d} T_{\text {II-L. }}$. It implies that although form II is not stable at lower pressures, above 
the I-II-L triple point, it possesses a stable domain at high temperature and high pressure. This is in fact similar to the pressure-temperature phase diagram of paracetamol, which also has a high-pressure phase and a monotropic system under ordinary pressure. ${ }^{6,7}$ The difference is that the slope of the I-II equilibrium of paracetamol is negative as a result of the higher density of the phase that is metastable under ordinary conditions. Thus, the Le Chatelier principle can be used in the case of paracetamol to foresee a possible phase transition by increased pressure. In the case of metacetamol, the phase stable under ordinary conditions is the densest polymorph, thus implying through the Le Chatelier principle that increasing pressure will in fact stabilize form I. Form II has a stable domain in the upper right-hand corner of the phase diagram just left of the liquid phase and this domain exists through a joint effect of heat and pressure. Therefore, only by determining the coordinates of the I-II-L triple point either by topological approach or by direct measurements under pressure can the existence of a stable domain for form II be demonstrated. APIs that possess a similar steep positive solid-solid equilibria in a monotropic system at ordinary pressure, which becomes enantiotropic at higher pressure are bicalutamide and fluoxetine nitrate. ${ }^{29,30}$ So far APIs with a negative I-II slope seem to occur as frequently, because next to paracetamol, also progesterone and ritonavir are known to exhibit this type of phase behavior. ${ }^{6,7,21,31}$ In a more general sense, systems that are monotropic under ordinary pressure, but become enantiotropic at higher pressure all belong to case three of the four pressure-temperature phase diagrams for dimorphism that have been described by Bakhuis-Roozeboom. ${ }^{28,32}$

From a more molecular mechanistic point of view, it can be said that the difference between the para and the meta position causes an inversion of the interaction behavior of the two molecules. Paracetamol's most stable polymorph under ambient conditions is the one with the lowest heat content, which implies that overall more energy is involved in its intermolecular interactions than in the case of the other, metastable polymorph. Nonetheless, the former has a lower density than the latter, thus the higher overall interaction energy does not translate into a denser structure. In the case of metacetamol, the polymorph stable under ambient conditions has the lowest heat content like paracetamol; however, in addition, it has the highest density, which in this case coincides with a higher intermolecular interaction energy. Nonetheless, as can be seen in Figure 4, the overall thermal expansion of form I seems to be somewhat larger than that of the less dense form II even if up to their melting points the specific volumes do not become equal. From this mechanistic point of view, the apparition of the second stability domain in metacetamol of a less dense, higher enthalpy form seems to be more logical than the phase diagram of paracetamol, where the form with the higher energy content is also the denser one (which causes the negative slope for the phase equilibrium between the two solid phases). Obviously, the interaction interplay in the case of these two isomers appears for now too subtle to be understood in detail, but it is a reason the more to use thermodynamics and take into account temperature and pressure to obtain consistent phase diagrams. 


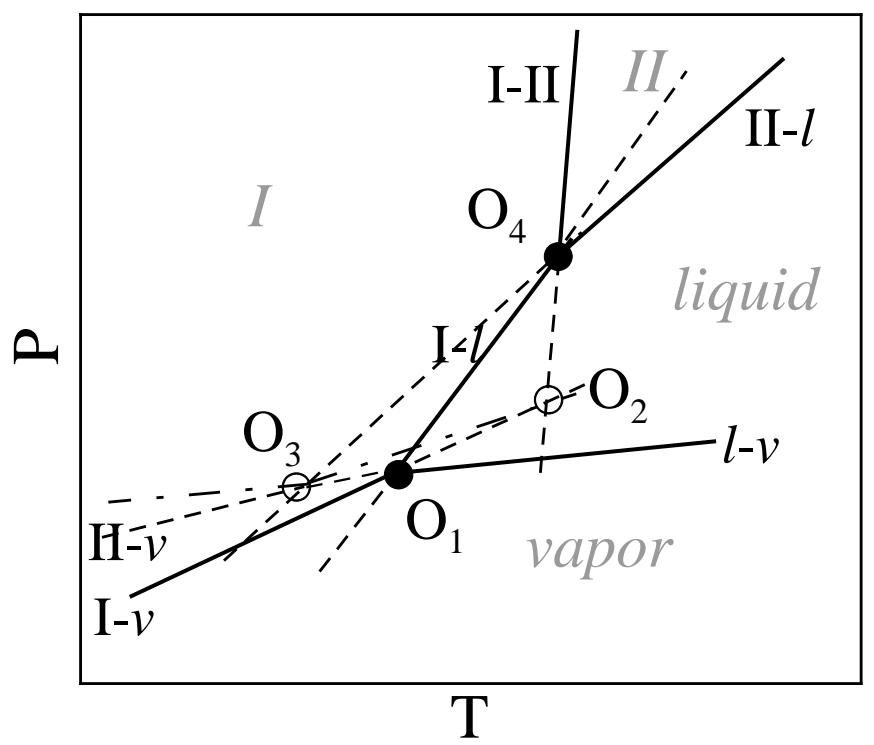

Figure 7. Topological pressure-temperature phase diagram of metacetamol. The stable phase domains in gray italic: form I, form II, the liquid and the vapor. Solid circles: stable triple points, $\mathrm{O}_{1}$ (I-L-V with $T=420.6 \mathrm{~K}$ and $P=5.2 \mathrm{~Pa}$ ) and $\mathrm{O}_{4}$ (I-II-L with $T=535 \mathrm{~K}$ and $P=692$ $\mathrm{MPa}$ ), whereas open circles are the metastable triple points $\mathrm{O}_{2}$ (I-II-V) and $\mathrm{O}_{3}$ (II-L-V). Thick solid lines: stable phase equilibria, broken lines: metastable equilibria, dotted lines: supermetastable equilibria. Pressure and temperature are not to scale.

\section{Conclusions}

Data present in the literature may lead to the conclusion that the specific volumes of the two polymorphs as a function of temperature cross each other. A careful experimental analysis on a single diffractometer has demonstrated that this is not the case and that form II has consistently a higher specific volume than form I. Thus it can be concluded that data from the literature should be considered cautiously, in particular because they may in general be obtained with different instruments and techniques.

The pressure-temperature phase behavior of metacetamol is an example of the third case of the four possible pressure-temperature phase diagrams of dimorphism described by Bakhuis-Roozeboom. ${ }^{28}$ The third case represents a monotropic relationship between two polymorphs that becomes enantiotropic at higher pressures. However, an important subtlety of this type of phase diagrams is the possibility for the solid-solid equilibrium curve to have a positive or a negative slope. In the case of a negative slope, as for paracetamol, the enantiotropy at higher pressure can be foreseen by application of the Le Chatelier principle. In the case of a positive slope, as for metacetamol, the density of the phase that becomes stable at high pressure is actually lower than that of the polymorph stable under ambient conditions and the enthalpy difference between the two polymorphs plays a decisive role in the appearance of the second polymorph. In the latter case, it is necessary to construct a topological pressure-temperature phase diagram to be able to differentiate between systems with overall monotropy and systems with a possible enantiotropy at higher pressures.

\section{Acknowledgements}

This work has been supported by the Spanish Ministry of Science and Innovation through the project FIS2014-54734-P and by the Generalitat de Catalunya under the project 2014 SGR581). 


\section{References}

1. McGregor L, Rychkov DA, Coster PL, Day S, Drebushchak VA, Achkasov AF, Nichol GS, Pulham CR, Boldyreva EV 2015. A new polymorph of metacetamol. CrystEngComm 17(32):6183-6192.

2. Hansen LK, Perlovich GL, Bauer-Brandl A 2006. N-(3-hydroxyphenyl)acetamide. Acta Crystallogr E 62:03627-03628.

3. Allouchi H, Nicolai B, Barrio M, Ceolin R, Mahe N, Tamarit J-L, Do B, Rietveld IB 2014. On the Polymorphism of L-Citrulline: Crystal Structure and Characterization of the Orthorhombic delta Form. Cryst Growth Des 14(3):1279-1286.

4. Barrio M, Maccaroni E, Rietveld IB, Malpezzi L, Masciocchi N, Céolin R, Tamarit J-L 2012. Pressure-temperature state diagram for the phase relationships between benfluorex hydrochloride forms I and II: A case of enantiotropic behavior. J Pharm Sci 101(3):1073-1078.

5. Rietveld IB, Barrio M, Tamarit J-L, Nicolaï B, Van de Streek J, Mahé N, Céolin R, Do B 2011. Dimorphism of the Prodrug L-Tyrosine Ethyl Ester: Pressure-Temperature State Diagram and Crystal Structure of Phase II. J Pharm Sci 100(11):4774-4782.

6. Espeau P, Céolin R, Tamarit JL, Perrin MA, Gauchi JP, Leveiller F 2005. Polymorphism of paracetamol: Relative stabilities of the monoclinic and orthorhombic phases inferred from topological pressure-temperature and temperature-volume phase diagrams. J Pharm Sci 94(3):524-539.

7. Ledru J, Imrie CT, Pulham CR, Céolin R, Hutchinson JM 2007. High pressure differential scanning Calorimetry investigations on the pressure dependence of the melting of paracetamol polymorphs I and II. J Pharm Sci 96(10):2784-2794.

8. Toscani S, Céolin R, Ter Minassian L, Barrio M, Veglio N, Tamarit J-L, Louër D, Rietveld IB 2016. Stability hierarchy between piracetam forms I, II, and III from experimental pressure-temperature diagrams and topological inferences. Int J Pharm 497:96-105.

9. Perlovich GL, Volkova TV, Manin AN, Bauer-Brandl A 2008. Influence of position and size of substituents on the mechanism of partitioning: A thermodynamic study on acetaminophens, hydroxybenzoic acids, and parabens. Aaps Pharmscitech 9(1):205216.

10. Gavezzotti A. 2013. Molecular Aggregation. Structure Analysis and Molecular Simulation of Crystals and Liquids. Oxford, UK: Oxford University Press. p 448.

11. Salud J, Barrio M, Lopez DO, Tamarit JL, Alcobe X 1998. Anisotropy of intermolecular interactions from the study of the thermal-expansion tensor. J Appl Crystallogr 31:748-757.

12. Jenau M, Reuter J, Tamarit JL, Wurflinger A 1996. Crystal and pVT data and thermodynamics of the phase transitions of 2-methyl-2-nitropropane. J Chem Soc Faraday T 92(11):1899-1904.

13. Barrio M, Tamarit JL, Negrier P, Pardo LC, Veglio N, Mondieig D 2008.

Polymorphism of CBr 2 Cl 2. New J Chem 32(2):232-239.

14. Parat B, Pardo LC, Barrio M, Tamarit JL, Negrier P, Salud J, López DO, Mondieig D 2005. Polymorphism of $\mathrm{CBrCl}_{3}$. Chem Mater 17(13):3359-3365.

15. Negrier P, Barrio M, Tamarit JL, Veglio N, Mondieig D 2010. Structure of Phase III and Polymorphism of $\left(\mathrm{CH}_{3}\right)_{3} \mathrm{CBr}$. Cryst Growth Des 10(6):2793-2800.

16. Negrier P, Barrio M, Tamarit JL, Pardo LC, Mondieig D 2012. Polymorphism in Halogen-Ethane Derivatives: CCl3-CF2Cl and CF3-CF2Cl. Cryst Growth Des 12(3):15131519. 
17. Negrier P, Tamarit JLI, Barrio M, Mondieig D 2013. Polymorphism in HalogenEthane Derivatives: CCl3-CCl3 and ClF2C-CF2Cl. Cryst Growth Des 13(2):782-791. 18. Tamarit JL, Lopez DO, Alcobe X, Barrio M, Salud J, Pardo LC 2000. Thermal and structural characterization of (CH3)(3)CCl. Chem Mater 12(2):555-563.

19. Negrier P, Pardo LC, Salud J, Tamarit JL, Barrio M, Lopez DO, Wurflinger A, Mondieig D 2002. Polymorphism of 2,2-dichloropropane: Crystallographic characterization of the ordered and disordered phases. Chem Mater 14(5):1921-1929. 20. Rietveld IB, Céolin R 2015. Phenomenology of crystalline polymorphism: overal monotropic behavior of the cardiotonic agent FK664 forms A and B. J Therm Anal Calorim 120(2):1079-1087.

21. Céolin R, Rietveld IB 2015. The topological pressure-temperature phase diagram of ritonavir, an extraordinary case of crystalline dimorphism. Ann Pharm Fr 73(1):2230 .

22. Nicolaï B, Rietveld IB, Barrio M, Mahé N, Tamarit J-L, Céolin R, Guéchot C, Teulon J-M 2013. Uniaxial negative thermal expansion in crystals of tienoxolol. Struct Chem 24(1):279-283.

23. Nicolaï B, Mahé N, Céolin R, Rietveld IB, Barrio M, Tamarit J-L 2011. Tyrosine alkyl esters as prodrug: the structure and intermolecular interactions of l-tyrosine methyl ester compared to l-tyrosine and its ethyl and n-butyl esters. Struct Chem 22(3):649-659.

24. Rodriguez-Carvajal J 1993. Recent advances in magnetic structure determination by neutron powder diffraction. Physica B 192:55-69.

25. Rodriguez-Carvajal J, Roisnel T, Gonzales-Platas J. 2005. Full-Prof suite version 2005. Laboratoire Léon Brillouin, CEA-CNRS, CEN Saclay, France.

26. Würflinger A 1975. Differential thermal-analysis under high-pressure IV. Lowtemperature DTA of solid-solid and solid-liquid transitions of several hydrocarbons up to 3 kbar. Ber Bunsen-Ges Phys Chem 79(12):1195-1201.

27. Riecke E 1890. Spezielle Fälle von Gleichgewichterscheinungen eines aus mehreren Phasen zusammengesetzten Systemes. Z Phys Chem (Munich) 6:411-429.

28. Bakhuis Roozeboom HW. 1901. Die heterogenen Gleichgewichte vom Standpunkte der Phasenlehre. Erstes Heft: Die Phasenlehre - Systeme aus einer Komponente., Braunschweig: Friedrich Vieweg und Sohn.

29. Gana I, Céolin R, Rietveld IB 2013. Bicalutamide polymorphs I and II: a monotropic phase relationship under ordinary conditions turning enantiotropic at high pressure. J Therm Anal Calorim 112(1):223-228.

30. Ceolin R, Rietveld I The topological pressure-temperature phase diagram of fluoxetine nitrate: monotropy unexpectedly turning into enantiotropy. Eur Phys J ST:submitted.

31. Barrio M, Espeau P, Tamarit JL, Perrin MA, Veglio N, Céolin R 2009.

Polymorphism of Progesterone: Relative Stabilities of the Orthorhombic Phases I and II Inferred from Topological and Experimental Pressure-Temperature Phase Diagrams. J Pharm Sci 98(5):1657-1670.

32. Céolin R, Rietveld IB 2016. X-ray crystallography, an essential tool for the determination of thermodynamic relationships between crystalline polymorphs. Ann Pharm Fr 74:12-20. 